\title{
Simplified Modelling and Control of a Synchronous Machine with Variable-Speed Six-Step Drive
}

\author{
Matthew K. Senesky*, Perry Tsao ${ }^{\dagger}$, Seth R. Sanders* \\ ${ }^{*}$ Dept. of Electrical Engineering and Computer Science, University of California, Berkeley, CA 94720 \\ ${ }^{\dagger}$ United Defense, L.P., Santa Clara, CA 95050
}

\begin{abstract}
We report on simple and intuitive techniques for the modelling of synchronous machines and their associated control systems. A scheme for control of electrical power flow is proposed for machines with variable-speed six-step drive. The scheme is developed with an eye toward efficient system operation, simple implementation, low latency in the control path and minimized cost of power electronics. Experimental results are presented for application of the control scheme to a homopolar inductor motor, as used in a flywheel energy storage system.
\end{abstract}

\section{INTRODUCTION}

Two techniques are presented here for simplification of the modelling and control design process for synchronous machines. What the two techniques have in common is a reliance on the underlying physical properties of the machine and drive system to suggest accurate yet tractable formulations of the control design problem. By reducing the order of the machine model and grouping control inputs and outputs according to their coupling strength, engineering intuition can be improved, and classical control results can be applied with confidence.

The first technique uses singular perturbation theory to perform a partitioning of the state-space model into "slow" and "fast" subsystems, which correspond to the mechanical and electrical variables, respectively. Since the electrical variables are often the focus of the control effort (e.g. in implementing torque or power control), the mechanical dynamics of the model can be suppressed. The second technique gives a methodology for determining whether a $2 \times 2$ multipleinput multiple-output (MIMO) system can be approximated as diagonal, in the sense that each input is primarily coupled to only one output and vice versa. If this is found to be the case, then an opportunity exists to independently synthesize a stabilizing scalar feedback loop for each input-output pair appearing on the diagonal, outside of the context of the overall system. Sufficient conditions exist to show that these control loops will then stabilize the overall system in the face of crosscoupling between the loops (i.e. nonzero off-diagonal terms). Further, the performance of the system will remain close to that of the diagonal approximation.

The motivation for this approach is the control of a flywheel energy storage system [1]. The system consists of a homopolar inductor motor/generator (HIM) whose rotor is also the flywheel energy storage element, and a six-step voltage source inverter fed from a DC bus. Application of the modelling and control design procedure described above to the HIM with six- step drive yields a simple and effective control of electrical power flow in the machine.

Cost is a driving factor in the design of such a system, and the power electronics associated with a variable-speed machine drive are a significant component of this cost. A sixstep drive scheme can reduce the cost of power electronics by enabling a closer matching of the DC bus voltage and the fundamental voltage waveform at the machine terminals than would be possible with a pulse width modulation (PWM) scheme. Six-step drive implies higher efficiency and higher maximum synchronous frequency than PWM as well.

\section{VARIABle-Speed Electric Machine Drives}

While the choice of drive for an electric machine may seem to be merely a detail of the hardware implementation, in fact it is critical to select a drive scheme before embarking on the system modelling and subsequent control design. Each drive type imposes unique constraints on the system inputs, and consequently the drive will determine which model formulations are convenient, and which are unsuitable.

By far the most common approach for variable-speed drives is PWM. However, six-step drive carries several advantages over PWM, particularly for high speed applications:

- Six-step drive generates a fixed amplitude excitation using the full bus voltage, resulting in semiconductor VA requirements that are closely matched to the inverter output. PWM schemes typically do not utilize the full bus voltage, but rather regulate the bus voltage down to avoid saturation of the duty cycle. The inverter semiconductor devices must still block the entire bus voltage however, and hence devices must be rated for a voltage higher than the intended output. Thus for a given amplitude of drive waveform, six-step allows the use of devices with lower VA ratings, which are less expensive.

- For six-step drive, the switching frequency of a given device is the same as the drive frequency. In contrast, PWM drive requires a large ratio of switching frequency to drive frequency, resulting in lower maximum drive frequencies for a given choice of semiconductor device. This also implies that for a given drive frequency, sixstep will exhibit lower switching dependent losses.

- For separately excited synchronous machines, running at unity power factor with six-step drive allows for zerocurrent switching, further reducing switching dependent 
losses. PWM requires hard switching, leading to associated losses and device stresses.

- Finally, six-step drive produces greatly reduced high frequency harmonics as compared to PWM, resulting in lower rotor and stator core losses [2].

\section{SYNCHRONOUS MACHINE MOdEL}

In a stationary reference frame, the standard two-axis model for the electrical dynamics of a synchronous machine can be written as

$$
\frac{d}{d t} \lambda_{a b f}=-\mathbf{R L}(\theta)^{-1} \lambda_{a b f}+V_{a b f}
$$

where $\lambda_{a b f}$ and $V_{a b f}$ indicate the vectors of flux linkages and terminal voltages, respectively, for armature windings $a$ and $b$ and field winding $f$. The term $\mathbf{R}$ is a diagonal matrix of winding resistances and $\mathbf{L}(\theta)$ is a symmetric inductance matrix dependent on the rotor angle $\theta$. Electrical torque $\tau_{e}$ is given by

$$
\tau_{e}=\frac{d}{d \theta}\left(\frac{1}{2} \lambda_{a b f}^{T} \mathbf{L}(\theta)^{-1} \lambda_{a b f}\right)
$$

and the mechanical speed of the rotor, $\omega_{m}$, is governed by

$$
\frac{d}{d t} \omega_{m}=\frac{1}{J}\left(\tau_{e}-B_{v} \omega_{m}\right)
$$

where $J$ represents rotor inertia and $B_{v}$ is a viscous drag term.

Equation 1 can be transformed into a synchronous reference frame denoted $(d, q)$, resulting in a time invariant model. The armature voltage vector is chosen to define the $q$ axis; as a consequence, for sinusoidal drives $V_{q}$ is constant and $V_{d}$ is identically zero.

The only choice of input for a conventional fixed voltage six-step drive is the drive frequency, hence $\omega_{e}$ is one system input. If a high bandwidth current control loop is implemented to set $i_{f}, \lambda_{f}$ drops out of the model, and $i_{f}$ appears as a second input. Thus the electrical dynamics simplify to

$$
\begin{aligned}
\frac{d}{d t} \lambda_{d} & =-\frac{R}{L} \lambda_{d}+\omega_{e} \lambda_{q}+\frac{R L_{m}}{L} i_{f} \cos \theta \\
\frac{d}{d t} \lambda_{q} & =-\omega_{e} \lambda_{d}-\frac{R}{L} \lambda_{q}-\frac{R L_{m}}{L} i_{f} \sin \theta+V_{q} \\
\frac{d}{d t} \theta & =\omega_{e}-\frac{N}{2} \omega_{m}
\end{aligned}
$$

where Eq. 6 describes the evolution of the angle $\theta$ between the $d$ and $f$ axes for an $N$-pole machine. Finally, outputs of interest are $i_{d}$ and $i_{q}$ :

$$
\begin{aligned}
& i_{d}=\frac{1}{L} \lambda_{d}-\frac{L_{m}}{L} i_{f} \cos \theta \\
& i_{q}=\frac{1}{L} \lambda_{q}+\frac{L_{m}}{L} i_{f} \sin \theta .
\end{aligned}
$$

Equations 4-8 can be linearized, yielding an LTI system of the form

$$
\begin{aligned}
\frac{d}{d t} x & =\mathbf{A} x+\mathbf{B} u \\
y & =\mathbf{C} x+\mathbf{D} u
\end{aligned}
$$

with state vector $x=\left[\begin{array}{llll}\lambda_{d} & \lambda_{q} & \theta & \omega_{m}\end{array}\right]^{T}$, input $u=\left[\begin{array}{ll}i_{f} & \omega_{e}\end{array}\right]^{T}$ and output $y=\left[\begin{array}{ll}i_{d} & i_{q}\end{array}\right]^{T}$. The interested reader will find the exact form of the Jacobian matrices $\mathbf{A}, \mathbf{B}, \mathbf{C}$ and $\mathbf{D}$ in Appendix I.
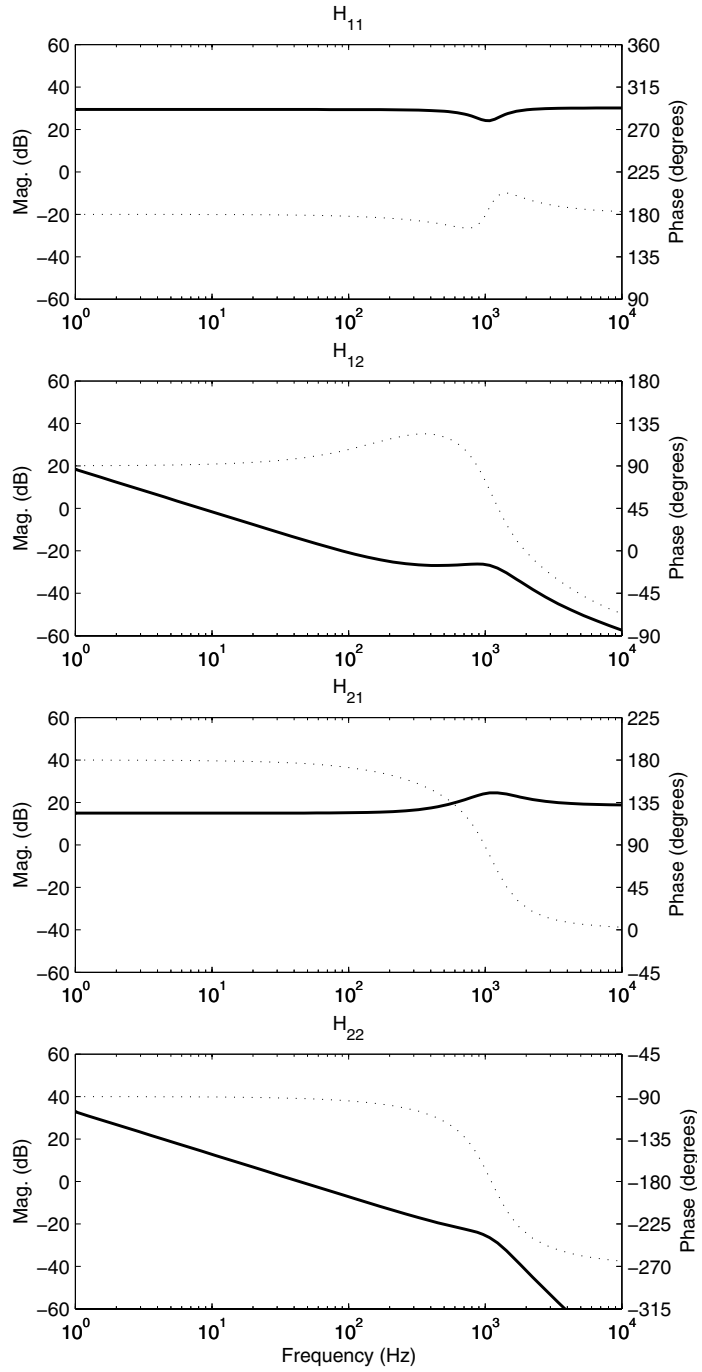

Fig. 1. Bode plots of synchronous machine electrical dynamics corresponding to Eqs. 12-15. Solid lines indicate magnitude, dotted lines indicate phase.

\section{Time-Scale Separation}

In electric machines, one expects intuitively that the time constants of the electrical variables will be much faster than those of the mechanical variables. Hence in dealing with electrical dynamics the speed of the rotor appears to be constant, while relative to the mechanical time constants electrical transients appear to settle instantaneously. The model given by Eqs. $9-10$ can thus be approximated by a partitioning into "fast" and "slow" subsystems.

Such a partitioning achieves a great simplification of the model at the expense of a slight error in the calculation of system dynamics. For example, the approximate two-timescale model of the experimental system discussed in Sec. VII estimates the system eigenvalues to within $10 \%$ of the values given by the original model. Singular perturbation theory provides a formal framework for such system partitioning. Relevant results are presented in Appendix II; for a detailed treatment of the theory, see [3]. 


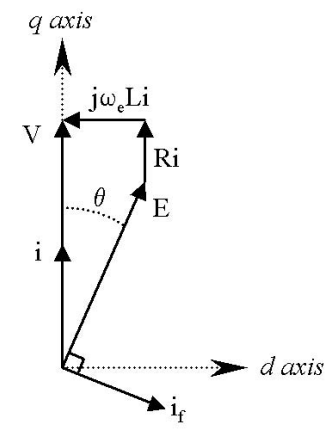

Fig. 2. Phasor diagram for a synchronous machine operating at unity power factor.

In implementing control of electrical rather than mechanical variables, the order of the model can be reduced such that the slow dynamic effects of the mechanical subsystem are suppressed. This approximation of the fast subsystem neglects the effect of the evolution of $\omega_{m}$. The reduced-order transfer matrix $\mathbf{H}$ for the synchronous machine model is thus

$$
\left[\begin{array}{l}
\hat{i}_{d} \\
\hat{i}_{q}
\end{array}\right]=\left[\begin{array}{ll}
H_{11} & H_{12} \\
H_{21} & H_{22}
\end{array}\right]\left[\begin{array}{c}
\hat{i}_{f} \\
\hat{\omega}_{e}
\end{array}\right]
$$

where a hat $\left(^{\wedge}\right)$ indicates a variable in the $s$-domain, and the transfer functions are given by

$$
\begin{aligned}
H_{11}= & -\left(\frac{L_{m}}{L} \cos \bar{\theta}\right)\left(s^{2}+\frac{R}{L} s+\bar{\omega}_{e}^{2}\right. \\
& \left.+\bar{\omega}_{e} \frac{R}{L} \tan \bar{\theta}\right) / D_{H} \\
H_{12}= & \left\{\bar{i}_{q} s^{2}+\left(\frac{R}{L} \bar{i}_{q}-\frac{1}{L} \bar{\omega}_{e} \bar{\lambda}_{d}\right) s+\bar{\omega}_{e}^{2}\left(\bar{i}_{q}-\frac{1}{L} \bar{\lambda}_{q}\right)\right. \\
& \left.+\bar{\omega}_{e} \frac{R}{L}\left(\bar{i}_{d}-\frac{1}{L} \bar{\lambda}_{d}\right)\right\} / s D_{H} \\
H_{21}= & \left(\frac{L_{m}}{L} \sin \bar{\theta}\right)\left(s^{2}+\frac{R}{L} s+\bar{\omega}_{e}^{2}-\bar{\omega}_{e} \frac{R}{L} \cot \bar{\theta}\right) / D_{H} \\
H_{22}= & \left\{-\bar{i}_{d} s^{2}-\left(\frac{R}{L} \bar{i}_{d}+\frac{1}{L} \bar{\omega}_{e} \bar{\lambda}_{q}\right) s-\bar{\omega}_{e}^{2}\left(\bar{i}_{d}-\frac{1}{L} \bar{\lambda}_{d}\right)\right. \\
& \left.+\bar{\omega}_{e} \frac{R}{L}\left(\bar{i}_{q}-\frac{1}{L} \bar{\lambda}_{q}\right)\right\} / s D_{H} \\
D_{H}= & s^{2}+2 \frac{R}{L} s+\left(\frac{R}{L}\right)+\bar{\omega}_{e}^{2} .
\end{aligned}
$$

The bar $\left(^{-}\right)$notation indicates the operating point value of a variable as used in linearizing the model. Bode plots of the transfer functions in Eqs. 12-15 are shown in Fig. 1.

\section{INDEPENDENT SCALAR FEEDBACK}

As a preliminary exercise to the discussion of control design, it is useful to examine the phasor diagram of electrical machine variables near unity power factor operation in Fig. 2. Classical analysis (as in [4]) of the figure suggests strong coupling for the diagonal (in the sense of Eq. 11) inputoutput pairs $\left(i_{f}, i_{d}\right)$ and $\left(\omega_{e}, i_{q}\right)$, and weak coupling for the off-diagonal pairs. To see this, assume that $R \ll \omega_{e} L$, and note that a change in the magnitude of $i_{f}$ results in a proportional change in the magnitude of $E$. This causes a change in the angle between $V$ and $i$ with little change in the magnitude of $i$. If the angle between $V$ and $i$ remains small (near unity

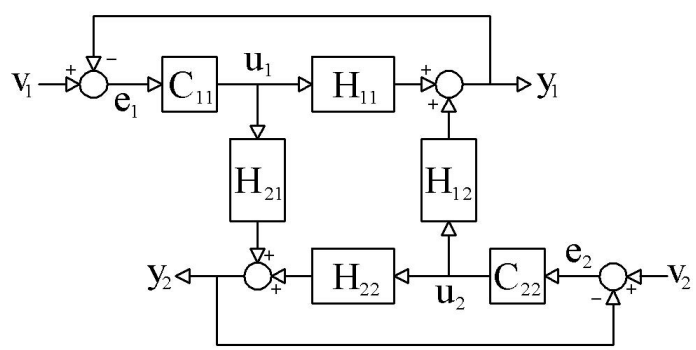

Fig. 3. Control system implementing feedback only for diagonal input-output pairs. $C_{i i}$ indicates a compensator, $H_{i j}$ indicates a plant block.

power factor), almost all the change in $i$ occurs along the $d$ axis. Similarly, varying $\omega_{e}$ will result in a slight difference between $\omega_{e}$ and $\frac{N}{2} \omega_{m}$, producing a change in the angle $\theta$. A change in $\theta$ primarily affects the magnitude of $i$ (rather than the angle of $i$ ), and hence most of the change in $i$ occurs along the $q$ axis. This qualitative analysis is supported by the bode plots in Fig. 1.

A quantitative analysis proceeds from Fig. 3, which shows a block diagram of the system in the $s$-domain. Because the development here is intended to be general, variables $e_{i}, u_{i}, v_{i}$, and $y_{i}, i=1,2$, are defined as in the figure. Note that feedback, with compensators denoted $C_{11}$ and $C_{22}$, is only implemented for the diagonal input-output pairs $\left(v_{i}, y_{i}\right)$. The following assumptions are made concerning the transfer functions in Fig. 3 [5]:

1) Each block represents a proper scalar transfer function of the form $N(s) / D(s)$.

2) No right half plane (RHP) pole-zero cancellations between $\left(C_{11} C_{22}\right)$ and $\left(H_{11} H_{22}\right)$ occur.

3) Any RHP poles that occur in $\left(H_{12} H_{21}\right)$ also occur (including multiplicity) in $\left(\mathrm{H}_{11} \mathrm{H}_{22}\right)$.

Consider independently the two single-input single-output (SISO) feedback systems formed by the blocks on the diagonal $\left(C_{11}, H_{11}, C_{22}\right.$, and $\left.H_{22}\right)$. Equivalently, assume $H_{12}=H_{21}=0$. Classical results from SISO control theory apply to these two systems, and given assumptions 1 and 2, internally stable closed-loop systems can be formed by proper design of the compensators $C_{11}$ and $C_{22}$. Disregarding inputs and outputs, the two SISO systems can be consolidated into equivalent closed-loop blocks $-C_{11} S_{1}$ and $-C_{22} S_{2}$, where

$$
\begin{aligned}
S_{1} & =\frac{1}{1+C_{11} H_{11}} \\
S_{2} & =\frac{1}{1+C_{22} H_{22}} .
\end{aligned}
$$

Now consider the system as a whole (i.e. lift the restriction that $\left.H_{12}=H_{21}=0\right)$. The stability of the overall system can be examined by breaking the loop between any two of the remaining four blocks, and finding the open-loop transfer function

$$
G=H_{12} H_{21} C_{11} S_{1} C_{22} S_{2} .
$$

The open-loop poles of $G$ are given by the poles of $S_{1}$ and $S_{2}$, and any stable poles of $H_{12}$ and $H_{21}$ not cancelled by 
$H_{11}$ and $H_{22}$. By assumption 3, any unstable poles of $H_{12}$ and $H_{21}$ are exactly cancelled by zeros of $S_{1}$ and $S_{2}$. Further, the poles of $S_{1}$ and $S_{2}$ have been explicitly placed for stability and performance via prudent design of $C_{11}$ and $C_{22}$. Thus the structure of $G$ and the assumptions above guarantee that $G$ has no unstable open-loop poles.

Note that any uncancelled poles of $H_{12}$ and $H_{21}$ are stable, but unaffected by either SISO loop closure. While this inability to place every pole represents a disadvantage of the diagonal control approach, a large subset of systems exists for which the poles of $H_{12}$ and $H_{21}$ are either acceptable or will cancel entirely.

Given that $G$ is guaranteed to be open-loop stable, the closed-loop stability of $G$ can be demonstrated by application of the small gain theorem [6]. Specifically, if the open-loop poles of $G$ are stable, and

$$
\|G\|_{\infty}<1
$$

then the closed-loop poles of $G$ are stable.

A slight manipulation of $G$ can improve intuition with respect to the condition given by Eq. 19. Substituting in Eqs. 16-17, and multiplying in both the numerator and denominator by the quantity $\left(H_{11} H_{22}\right)$ gives

$$
G=\frac{H_{12} H_{21}}{H_{11} H_{22}} \cdot \frac{C_{11} H_{11}}{1+C_{11} H_{11}} \cdot \frac{C_{22} H_{22}}{1+C_{22} H_{22}} .
$$

Defining the terms

$$
\begin{aligned}
\Delta & =\frac{H_{12} H_{21}}{H_{11} H_{22}} \\
T_{1} & =\frac{C_{11} H_{11}}{1+C_{11} H_{11}} \\
T_{2} & =\frac{C_{22} H_{22}}{1+C_{22} H_{22}}
\end{aligned}
$$

the small gain condition for stability becomes

$$
\left\|\Delta T_{1} T_{2}\right\|_{\infty}<1
$$

or equivalently

$$
\max _{0<j \omega<\infty}\left\{|\Delta| \cdot\left|T_{1}\right| \cdot\left|T_{2}\right|\right\}<1 .
$$

The condition given by Eq. 25 implies an elegant design methodology for stabilizing $2 \times 2$ MIMO systems. (We note the similarity to "individual channel design" described extensively in [7], [8], [9], [10], [11]). The magnitude of the term $\Delta$ gives a measure of the "diagonal-ness" of the system that is, a measure of the relative coupling strengths of diagonal versus off-diagonal input-output pairs. If the magnitude of $\Delta$ is much less than unity over the desired control bandwidth, then chances are good that the system can be stabilized by two independently designed feedback loops. (As a corollary, a magnitude much larger than unity suggests that exchanging the input-output pairs will result in favorable conditions for independent control.) It is then a straightforward task to design compensators $C_{11}$ and $C_{22}$ to meet given specifications for

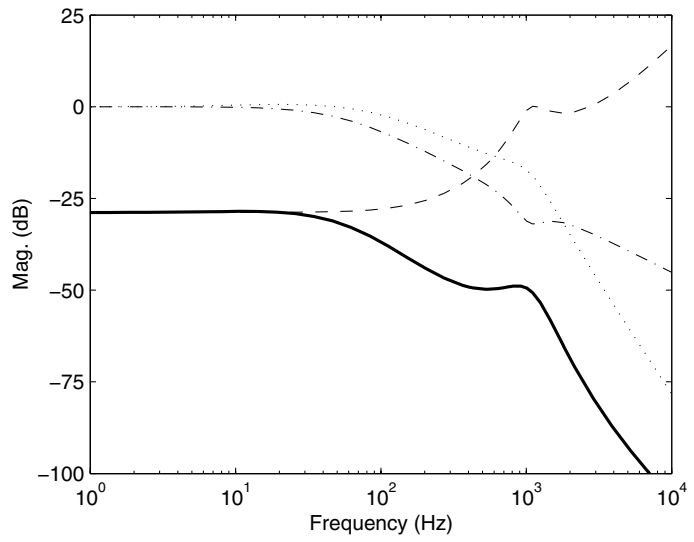

Fig. 4. Plot of $|\Delta|$ (dashed), $\left|T_{1}\right|$ (dash-dotted), $\left|T_{2}\right|$ (dotted), and $|\Delta| \cdot\left|T_{1}\right| \cdot\left|T_{2}\right|$ (solid).

the two independent SISO systems. If each SISO system can be stabilized and made to exhibit a suitably well-damped response, then the MIMO system is guaranteed to be stable as well. Consider Fig. 4, which uses values from the experimental system of Sec. VII. The figure makes explicit the bandwidth limitations imposed by the structure of the plant. Examining the plot of $|\Delta|$, it is clear that well-damped closed-loop transfer functions $T_{1}$ and $T_{2}$, designed to have approximately the same corner frequency as $\Delta$, will satisfy Eq. 25 .

Note that the small gain theorem gives a sufficient, but not necessary, condition for stability of the closed-loop system. A necessary and sufficient condition is given by the Nyquist theorem [12]. Compared to the Nyquist theorem, the small gain theorem is overly restrictive; Nyquist design constraints permit open-loop gain to exceed unity for phase angles that are far from $180^{\circ}$.

However, the restriction imposed by the small gain theorem proves to be beneficial, in that it also keeps closed-loop performance close to the performance predicted by the independent design of $C_{11}$ and $C_{22}$. Equations 26-29 give the closed-loop transfer functions for Fig. 3. The transfer functions have been arranged such that in each case the first term represents what might be called the "decoupled" result, while the term in parentheses represents a multiplicative perturbation resulting from loop interactions via off-diagonal terms.

$$
\begin{aligned}
\frac{y_{1}}{v_{1}} & =\frac{C_{11} H_{11}}{1+C_{11} H_{11}}\left(\frac{1-\Delta T_{2}}{1-\Delta T_{1} T_{2}}\right) \\
\frac{y_{1}}{v_{2}} & =C_{22} H_{12}\left(\frac{S_{1} S_{2}}{1-\Delta T_{1} T_{2}}\right) \\
\frac{y_{2}}{v_{1}} & =C_{11} H_{21}\left(\frac{S_{1} S_{2}}{1-\Delta T_{1} T_{2}}\right) \\
\frac{y_{2}}{v_{2}} & =\frac{C_{22} H_{22}}{1+C_{22} H_{22}}\left(\frac{1-\Delta T_{1}}{1-\Delta T_{1} T_{2}}\right)
\end{aligned}
$$

Bode plots of Eqs. 26-29 using values from the experimental system of Sec. VII are presented in Fig. 5. For each equation, the figure shows the "decoupled" term (which is the 
(a)

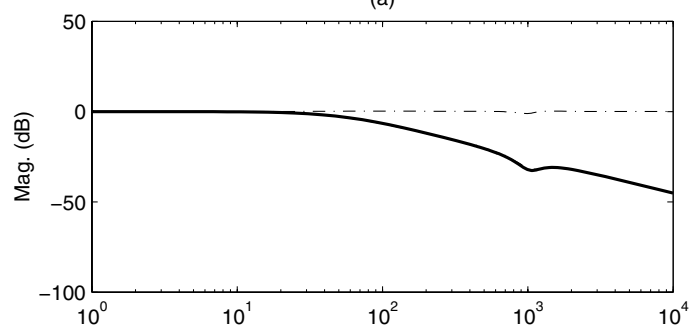

(b)

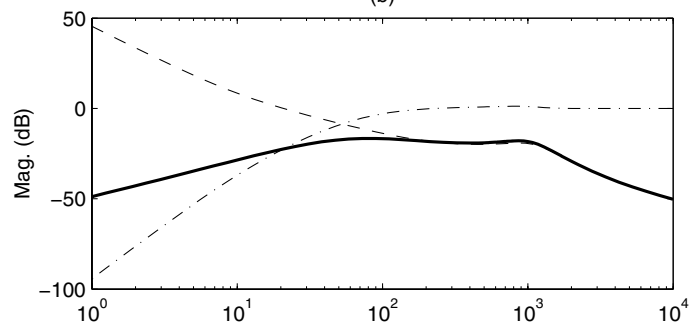

(c)

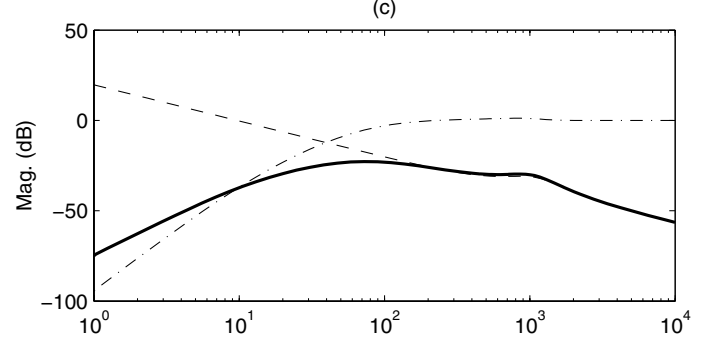

(d)

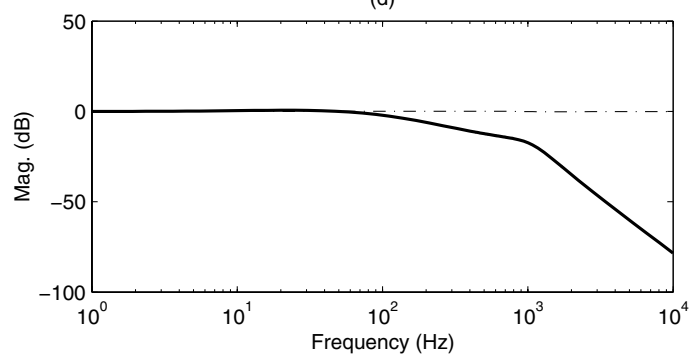

Fig. 5. Bode plots of (a) Eq. 26, (b) Eq. 27, (c) Eq. 28, (d) Eq. 29. Dashed lines indicate decoupled terms, dash-dotted lines indicate perturbation terms, solid lines indicate combined results. Note that the solid and dashed lines in (a) and (d) coincide up to the resolution of the figure.

result obtained by considering each transfer function independently), the "coupling" term (the term in parentheses, which represents a multiplicative perturbation resulting from offdiagonal coupling), and the combined result. The perturbation of the diagonal terms is close to unity over a wide range of frequencies, preserving the performance of the SISO loop designs. The perturbation of the off-diagonal terms serves to increase the decoupling of the two control loops over the control bandwidth, improving upon the original assumption of weak coupling.

\section{Control Strategy}

Given the analysis of Sec. V, it is clear that the tracking of commands for $i_{d}$ and $i_{q}$ in the synchronous machine can be achieved with two independent scalar feedback loops.

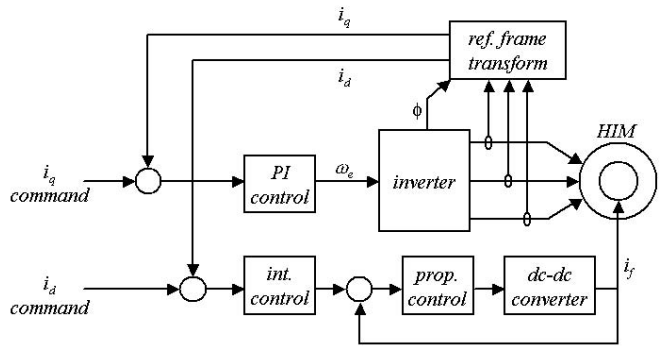

Fig. 6. Control system block diagram.

Implementation of this scheme is shown in Fig. 6.

An attractive feature of the control scheme is its simplicity in both architecture and implementation. The reference frame is defined by the inverter voltage, hence the reference frame angle ( $\phi$ in Fig. 6) is explicitly known six times per period of the electrical frequency - every time that the inverter switches. By sampling armature currents at these instants, sampled current values can be transformed into the rotating reference frame and compared to commands. Unlike fluxoriented vector-based control schemes, no observer is required to resolve the reference frame, and no sampling of terminal voltages is required. Scalar control laws - an integrator for $C_{11}$ and proportional-integral (PI) form for $C_{22}$ - yield the desired response characteristics. Because only a small number of control calculations need to be performed, latency in the control path is small. Assuming a six-step drive operating from a fixed bus voltage, this current control amounts to instantaneous control of active and reactive power in the machine.

\section{IMPLEMENTATION AND RESULTS}

The above control scheme was implemented on a homopolar inductor machine (a separately excited synchronous machine) with six-step voltage source inverter drive. The machine is part of a flywheel energy storage system in which the motor/generator rotor also serves as the energy storage element. The system is described in detail in [1], [13], [14].

Figures 7-11 show system response to step commands of $i_{q}$ from $-80 A$ to $80 A$ and from $80 A$ to $-80 A$, while $i_{d}$ is commanded to a constant zero. Bus voltage was $70 \mathrm{~V}$, and rotor speed ranged from $15 \mathrm{krpm}$ at low-to-high $i_{q}$ transitions to $30 \mathrm{krpm}$ at high-to-low $i_{q}$ transitions.

Figure 7 shows experimental data for electrical frequency and real current. However, since the machine is synchronous and the bus voltage is fixed, the figure can be thought of as showing flywheel speed and power flow in the machine. When the current (power) is positive, the machine speeds up, storing energy. Similarly, when current is negative, the machine slows down, returning its stored kinetic energy to the bus. Note the small transients that occur at each peak and valley of the $\omega_{e}$ trajectory. These represent instantaneous departures from synchronous operation to change the angle between reference frame and rotor. 

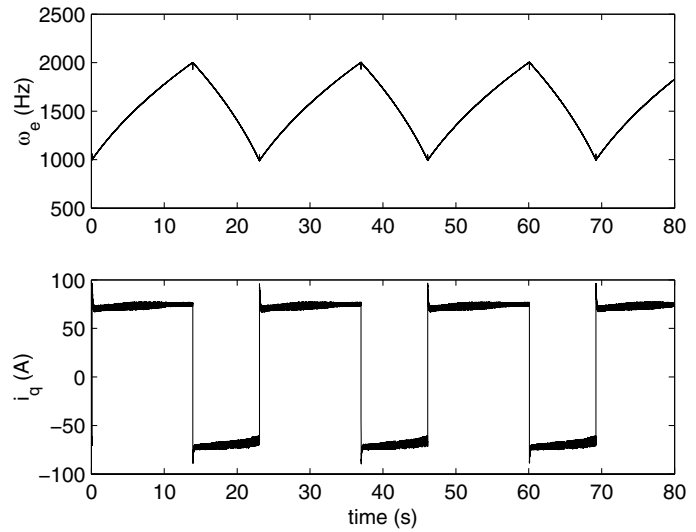

Fig. 7. Experimental response to step $i_{q}$ commands, showing $\omega_{e}$ (top) and $i_{q}$ (bottom)
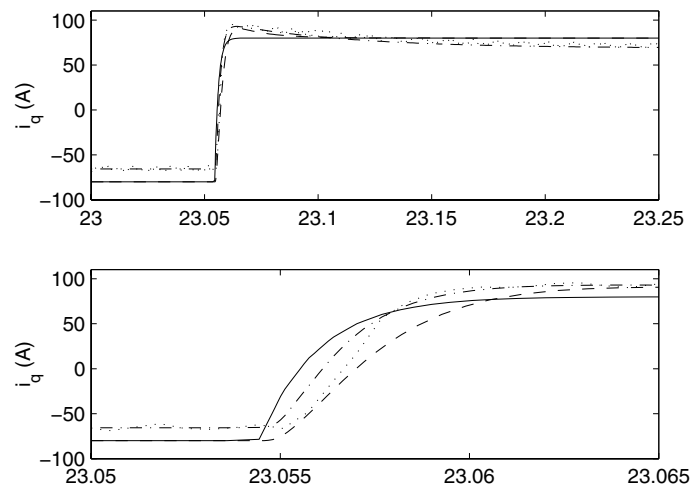

Fig. 8. Step response of $i_{q}$, for an $i_{q}$ step command from $-80 A$ to $80 A$ and $i_{d}$ command of constant zero, showing $i_{q}$ command (solid), experimental result (dotted), nonlinear model simulation (dashed), and linear model simulation (dash-dotted). The lower figure shows the plot from the upper figure in an expanded time scale.

Figures 8-11 focus on the transients, showing the $i_{d}$ and $i_{q}$ commands, experimental $i_{d}$ and $i_{q}$ outputs, simulations using the nonlinear dynamics presented in Eqs. 2-8 and simulations using the linear reduced-order system Eqs. 12-15. Note that the $i_{q}$ command is not a pure step input, but rather an exponential rise with very short time constant. This input was used in the experimental system to reduce sharp transient spikes, and hence simulations were performed with the same input.

The nonlinear model shows excellent agreement with the experimental results, except for sharp transients on $i_{d}$. These were caused by fluctuations in the bus voltage not included in the model. The linear model accurately captures the rise and fall times of the output currents, although it fails to predict some overshoot and steady-state error.

\section{CONCLUSIONS}

Analysis of a synchronous machine and variable-speed drive system was performed, and insights were provided as to useful simplifications that aid the control design process. A control scheme was presented that permits six-step inverter operation, which has several advantages over PWM. The
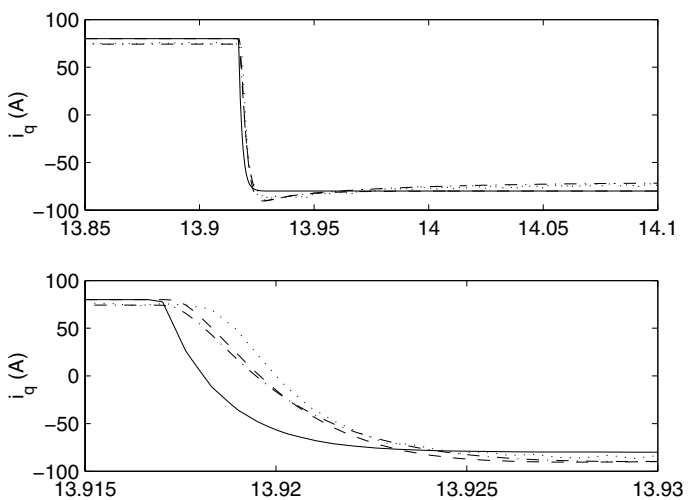

Fig. 9. Step response of $i_{q}$, for an $i_{q}$ step command from $80 A$ to $-80 A$ and $i_{d}$ command of constant zero, showing $i_{q}$ command (solid), experimental result (dotted), nonlinear model simulation (dashed), and linear model simulation (dash-dotted). The lower figure shows the plot from the upper figure in an expanded time scale.
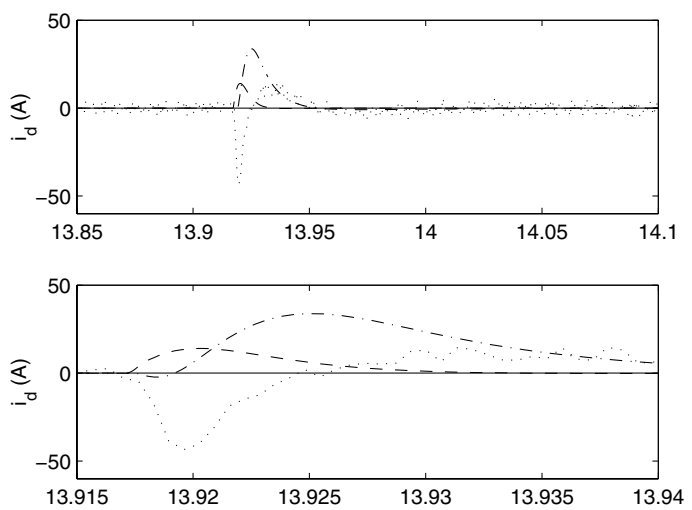

Fig. 10. Step response of $i_{d}$, for an $i_{q}$ step command from $-80 A$ to $80 A$ and $i_{d}$ command of constant zero, showing $i_{d}$ command (solid), experimental result (dotted), nonlinear model simulation (dashed), and linear model simulation (dash-dotted). The lower figure shows the plot from the upper figure in an expanded time scale.
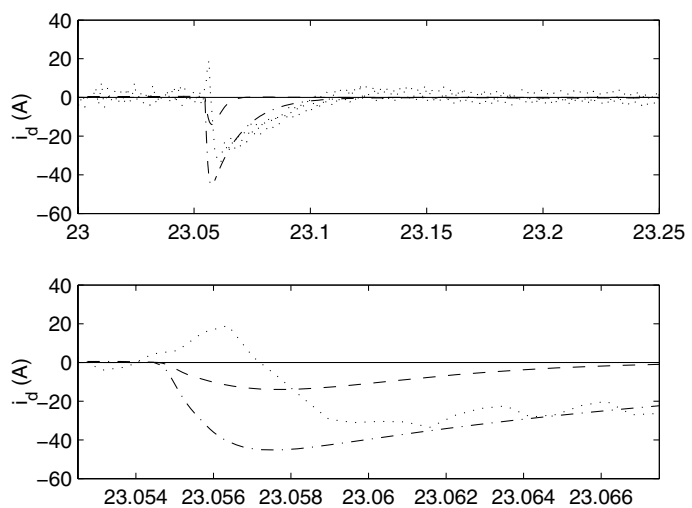

Fig. 11. Step response of $i_{d}$, for an $i_{q}$ step command from $80 A$ to $-80 A$ and $i_{d}$ command of constant zero, showing $i_{d}$ command (solid), experimental result (dotted), nonlinear model simulation (dashed), and linear model simulation (dash-dotted). The lower figure shows the plot from the upper figure in an expanded time scale. 
feasibility of the control scheme, as well as the usefulness of the design methodology, was demonstrated through application to experimental hardware.

\section{APPENDIX I}

The Jacobians A, B, C and $\mathbf{D}$ described in Sec. III are given by Eqs. 30-33. The bar $\left(^{-}\right)$notation indicates the operating point value of a variable as used in linearizing the model.

$$
\begin{aligned}
& \mathbf{A}=\left[\begin{array}{c|c|c||c}
-\frac{R}{L} & \bar{\omega}_{e} & -\frac{R L_{m}}{L} \bar{i}_{f} \sin \bar{\theta} & 0 \\
\hline-\bar{\omega}_{e} & -\frac{R}{L} & -\frac{R L_{m}}{L} \bar{i}_{f} \cos \bar{\theta} & 0 \\
\hline 0 & 0 & 0 & -\frac{N}{2} \\
\hline \hline a_{41} & a_{42} & a_{43} & -\frac{B_{v}}{J}
\end{array}\right] \\
& a_{41}=\frac{N}{2} \frac{1}{J} \frac{L_{m}}{L} \bar{i}_{f} \sin \bar{\theta} \\
& a_{42}=\frac{N}{2} \frac{1}{J} \frac{L_{m}}{L} \bar{i}_{f} \cos \bar{\theta} \\
& a_{43}=\frac{N}{2} \frac{1}{J} \frac{L_{m}}{L} \bar{i}_{f}\left(\bar{\lambda}_{d} \cos \bar{\theta}-\bar{\lambda}_{q} \sin \bar{\theta}\right) \\
& \mathbf{B}=\left[\begin{array}{c|c}
\frac{R L_{m}}{L} \cos \bar{\theta} & \bar{\lambda}_{q} \\
\hline-\frac{R L_{m}}{L} \sin \bar{\theta} & -\bar{\lambda}_{d} \\
\hline 0 & 1 \\
\hline \hline \frac{N}{2} \frac{1}{J} \frac{L_{m}}{L}\left(\bar{\lambda}_{d} \sin \bar{\theta}+\bar{\lambda}_{q} \cos \bar{\theta}\right) & 0
\end{array}\right] \\
& \mathbf{C}=\left[\begin{array}{c|c|c||c}
\frac{1}{L} & 0 & \frac{L_{m}}{L} \bar{i}_{f} \sin \bar{\theta} & 0 \\
\hline 0 & \frac{1}{L} & \frac{L_{m}}{L} \bar{i}_{f} \cos \bar{\theta} & 0
\end{array}\right] \\
& \mathbf{D}=\left[\begin{array}{c|c}
-\frac{L_{m}}{L} \cos \bar{\theta} & 0 \\
\hline \frac{L_{m}}{L} \sin \bar{\theta} & 0
\end{array}\right]
\end{aligned}
$$

\section{APPENDIX II}

Here results from singular perturbation analysis are developed. Considering Eqs. 9 and 10, the system is partitioned according to the double lines in Eqs. 30-33, where $x_{f}=\left[\begin{array}{lll}\lambda_{d} & \lambda_{q} & \theta\end{array}\right]^{T}$ and $x_{s}=\omega_{m}$. A small parameter $\varepsilon>0$ explicitly indicates that $x_{f}$ changes quickly with respect to $x_{s}$, such that

$$
\begin{aligned}
\varepsilon \frac{d}{d t} x_{f} & =\mathbf{A}_{11} x_{f}+\mathbf{A}_{12} x_{s}+\mathbf{B}_{1} u \\
\frac{d}{d t} x_{s} & =\mathbf{A}_{21} x_{f}+\mathbf{A}_{22} x_{s}+\mathbf{B}_{2} u .
\end{aligned}
$$

An invertible change of variables can be defined such that $w_{f, s}=$ $\mathbf{M} x_{f, s}$, where $\mathbf{M}$ is given by

$$
\mathbf{M}=\left[\begin{array}{cc}
\mathbf{I}_{f} & \mathbf{M}_{12} \\
0 & \mathbf{I}_{s}
\end{array}\right] .
$$

Applying $\mathbf{M}$ to Eqs. $34-35$ gives

$$
\begin{aligned}
\varepsilon \frac{d}{d t} w_{f}= & \left(\mathbf{A}_{11}+\varepsilon \mathbf{M}_{12} \mathbf{A}_{21}\right) w_{f}+f\left(\mathbf{M}_{12}, \varepsilon\right) w_{s} \\
& +\left(\mathbf{B}_{1}+\varepsilon \mathbf{M}_{12} \mathbf{B}_{2}\right) u \\
\frac{d}{d t} w_{s}= & \mathbf{A}_{21} w_{f}+\left(\mathbf{A}_{22}-\mathbf{A}_{21} \mathbf{M}_{12}\right) w_{s}+\mathbf{B}_{2} u
\end{aligned}
$$

where

$$
\begin{aligned}
f\left(\mathbf{M}_{12}, \varepsilon\right)= & \mathbf{A}_{12}-\mathbf{A}_{11} \mathbf{M}_{12}+\varepsilon \mathbf{M}_{12} \mathbf{A}_{22} \\
& -\varepsilon \mathbf{M}_{12} \mathbf{A}_{21} \mathbf{M}_{12} .
\end{aligned}
$$

From here, a block-triangular form can be obtained by finding a value of $\mathbf{M}_{12}$ such that $f\left(\mathbf{M}_{12}, \varepsilon\right)=0$. This is possible only if $\mathbf{A}_{11}^{-1}$ exists. Assuming this is the case, $\mathbf{M}_{12}$ can be found via a Taylor series expansion, giving

$$
\mathbf{M}_{12}(\varepsilon)=\mathbf{A}_{11}^{-1} \mathbf{A}_{12}+\varepsilon \mathbf{A}_{11}^{-2} \mathbf{A}_{12} \mathbf{A}_{0}+O\left(\varepsilon^{2}\right)
$$

where $\mathbf{A}_{0}=\mathbf{A}_{22}-\mathbf{A}_{21} \mathbf{A}_{11}^{-1} \mathbf{A}_{12}$. Hence the slow subsystem is governed by

$$
\begin{aligned}
\frac{d}{d t} w_{s}(t)= & \mathbf{A}_{21} w_{f}(t)+\left(\mathbf{A}_{22}-\mathbf{A}_{21} \mathbf{M}_{12}(\varepsilon)\right) w_{s}(t) \\
& +\mathbf{B}_{2}(u)
\end{aligned}
$$

and the fast subsystem is governed by

$$
\begin{aligned}
\frac{d}{d \tau} w_{f}(\tau)= & \left(\mathbf{A}_{11}+\varepsilon \mathbf{M}_{12} \mathbf{A}_{21}\right) w_{f}(\tau) \\
& +\left(\mathbf{B}_{1}+\varepsilon \mathbf{M}_{12} \mathbf{B}_{2}\right) u(\tau)
\end{aligned}
$$

where the time scale for the fast dynamics has been "stretched" by defining $\tau=\frac{t-t_{o}}{\varepsilon}$. Notice that as $\varepsilon \rightarrow 0$, Eq. 42 approaches

$$
\frac{d}{d \tau} w_{f}(\tau)=\mathbf{A}_{11} w_{f}(\tau)+\mathbf{B}_{1} u(\tau)
$$

which is the fast subsystem that one intuitively expects. Further, Eq. 41 approaches

$$
\begin{aligned}
\frac{d}{d t} w_{s}(t)= & \mathbf{A}_{21} w_{f}(t)+\left(\mathbf{A}_{22}-\mathbf{A}_{21} \mathbf{A}_{11}^{-1} \mathbf{A}_{12}\right) w_{s}(t) \\
& +\mathbf{B}_{2}(u)
\end{aligned}
$$

giving an approximation of the slow subsystem.

It can be shown for Eq. 43 that if all the eigenvalues of $\mathbf{A}_{11}$ (nonzero by previous assumption) have negative real parts, then $w_{f}(\tau)$ converges exponentially to a finite solution dependent on $u(\tau)$. Hence a necessary requirement for a stable reduced-order system is

$$
R e\left(\lambda_{i}\left(\mathbf{A}_{11}\right)\right)<-c<0 \quad \forall i
$$

where $\lambda_{i}\left(\mathbf{A}_{11}\right)$ indicates the $i t h$ eigenvalue of $\mathbf{A}_{11}$, and $c>0$.

This condition is obviously not met for the $\mathbf{A}_{11}$ matrix given in Eq. 30); in fact this matrix is singular. However, it is possible to satisfy the condition given by Eq. 45 by applying feedback control to the fast subsystem. Hence the modelling effort may still proceed with the reduced-order system given by $\mathbf{A}_{11}, \mathbf{B}_{1}, \mathbf{C}_{1}$, and $\mathbf{D}$.

\section{REFERENCES}

[1] P. Tsao, M. Senesky, and S. Sanders, "An integrated flywheel energy storage system with homopolar inductor motor/generator and highfrequency drive," IEEE Trans. Ind. Applicat., vol. 39, pp. 1710-1725, Nov./Dec. 2003.

[2] A. Boglietti, P. Ferraris, M. Lazzari, and F. Profumo, "Energetic behavior of soft magnetic materials in the case of inverter supply," IEEE Trans. Ind. Applicat., vol. 30, pp. 1580-1587, Nov. 1994.

[3] P. Kokotovic, H. Khalil, and J. O'Reilly, Singular Perturbation Methods in Control: Analysis and Design. Academic Press, 1986.

[4] A. Bergen and V. Vittal, Power Systems Analysis. Prentice-Hall, 2000.

[5] J. Doyle, B. Francis, and A. Tannenbaum, Feedback Control Theory. MacMillan, 1992

[6] C. Desoer and M. Vidyasagar, Feedback Systems: Input-Output Properties. Academic Press, 1975.

[7] J. O'Reilly and W. E. Leithead, "Multivariable control by 'individual channel design'," International Journal of Control, vol. 54, pp. 1-46, July 1991.

[8] W. E. Leithead and J. O'Reilly, "Performance issues in the individual channel design of 2-input 2-output systems - i. structural issues," International Journal of Control, vol. 54, pp. 47-82, July 1991.

[9] _ - "Performance issues in the individual channel design of 2-input 2output systems - ii. robustness issues," International Journal of Control, vol. 55, pp. 3-47, Jan. 1992

[10] — "Performance issues in the individual channel design of $2-$ input 2-output systems - iii. non-diagonal control and related issues," International Journal of Control, vol. 55, pp. 265-312, Feb. 1992.

[11] — , "m-input m-output feedback control by individual channel design," International Journal of Control, vol. 56, pp. 1347-1397, Dec. 1992.

[12] H. Nyquist, "Regeneration theory," Bell System Tech. J., vol. 11, pp. 126-47, 1932

[13] M. Senesky, "Control of a synchronous homopolar machine for flywheel applications," Master's thesis, University of California, Berkeley, 2003.

[14] P. Tsao, "A homopolar inductor motor/generator and six-step drive flywheel energy storage system," Ph.D. dissertation, University of California, Berkeley, 2003. 